\title{
Review of: "AUY922 induces retinal toxicity through attenuating TRPM1"
}

NIKHIL AGRAWAL ${ }^{1}$

1 State University of New York at Buffalo

Potential competing interests: The author(s) declared that no potential competing interests exist.

This is an excellent manuscript and highlighting the retinal toxicity through attenuating TRPM1. 